\title{
Sliding mode control for slosh-free motion using a nonlinear sliding surface
}

\author{
Shailaja Kurode, Member, IEEE, Sarah Spurgeon, Senior Member, IEEE, B. Bandyopadhyay, Senior \\ Member, IEEE, and P. S. Gandhi, Member, IEEE,
}

\begin{abstract}
A new nonlinear switching surface is proposed for the design of a sliding mode controller for a class of mismatched uncertain systems. A control system for slosh-free motion of a partially filled liquid container is sought. This is representative of a broader class of systems to which the proposed methodology is applicable. A fundamental mode of lateral slosh is considered. A simple pendulum model is used to represent the lateral slosh. Given the difficulty of measuring the slosh states directly, a sliding mode observer is used for implementation of the resulting sliding mode control. The effectiveness of the theoretical developments is demonstrated via both simulation and experimental results.
\end{abstract}

Index Terms-Slosh; Sliding mode control; Nonlinear sliding surface

\section{INTRODUCTION}

Any motion of a liquid in a partially filled container is called slosh. Sloshing liquid induces additional forces and moments which affect the performance. Control of liquid sloshing has received sustained interest among researchers over the last four decades. The interaction of the slosh dynamics with the control system has a direct impact on both vehicle stability and performance [1]. Detrimental effects of liquid sloshing are experienced in several areas including space launch vehicles and satellites. Liquid sloshing in packaging industries may lead to improper sealing which further affects the shelf life. Slosh induced forces and moments may result in dangerous overturn of large liquid cargo. In space launch vehicles and long range missiles, liquid fuel weight is more than $70 \%$ of the total weight. The impact of liquid sloshing is therefore severe. Inadequate slosh suppression has resulted several mission failure. Control of slosh is thus an important problem of practical significance.

Measurement of slosh states for feedback is difficult. Various sensors have been reported in the literature, but their mounting, accuracy and cost are critical issues. Therefore passive techniques are commonly used for slosh suppression. Passive elements such as slosh absorbers and baffles are used

Manuscript received on December 18, 2009; Accepted for publication on Dec 15, 2011. The material in this paper was partially presented at the International Conference ECC 2009 at Budapest Hungary during Aug 23$26,2009$.

Shailaja Kurode is with Department of Electrical Engineering College of Engineering Pune, 411005 INDIA e-mail: srk.coep@gmail.com Sarah Spurgeon is with Department of Electronics (now the School of Engineering and Digital Arts) at the University of Kent U.K.email:s.k.spurgeon@kent.ac.uk B. Bandyopadhyay is with IDP in Systems and Control Engineering, Indian Institute of Technology Bombay, Mumbai 400076 INDIA e-mail: (see http://www.sc.iitb.ac.in/ bijnan)P. S. Gandhi is with Department of Mechanical engineering, Indian Institute of Technology Bombay, Mumbai 400076 INDIA e-mail: (see http://www.me.iitb.ac.in/ gandhi) to absorb slosh energy. Extensive analytical and experimental studies have been reported. However, use of passive elements adds weight, construction time and cost. This motivates control researchers to seek a suitable, cost effective advanced control implementation. Improved methods of prediction, analysis, and control of slosh have been researched across the world with each research group focussing on a particular application. In [2], an IIR filter was used to preshape the acceleration profile to obtain slosh-free motion of an open container. Grundelius et al. [3] considered the slosh problem in the packaging industry. The authors solved the problem by deriving a simplified mathematical model of the slosh phenomenon and applying optimal control techniques and iterative learning control to determine the appropriate acceleration reference. Yano et al. [4], [5] considered the slosh problem in the casting and steel industries. They used $H_{\infty}$ control to follow a trajectory which was designed using optimization techniques subject to a number of constraints including minimum slosh.

Very few active control methods have been reported in the literature see for example [6] and [7]. Venugopal and Bernstein in [6] used active control. They used two methods to investigate the slosh problem. In the first method they achieved control of slosh by controlling the pressure on the surface of liquid by speaker mounted on the top. The region above the surface was considered as a closed acoustic duct. Feedback control using LQG synthesis has been used to control slosh by varying pressure. The second method includes the use of a flap actuator on the surface. A flap was hinged at one end of the tank. The fluid flap system was modeled in state space. LQG synthesis was used. The slosh has been controlled by controlling the angular velocity of the flap. The flap or the speaker adds weight to the system. Moreover LQG synthesis is not fully robust. In [7], Gandhi et al. developed an active control strategy to stabilize slosh in a cylindrical tank using translational excitation as the control input. The control law was based on a Lyapunov approach and implemented using force feedback.

Modeling of slosh is potentially complex. Much of the aforementioned literature considers slosh modeled by a relatively simple mechanical model to simplify the control design. However, simpler models lead to inevitable plantmodel uncertainty and hence robustness issues. Sliding mode control (SMC) is known to be robust [8] and can be used to yield robust performance against model inaccuracy and uncertainties. Adaptive control strategies are emerging but require knowledge of the model structure whilst robust control methods offer robust performance against model inaccuracies 
[9] [10] [11].

Sliding mode control is one of the best known effective robust control methods for nonlinear uncertain systems. This elegant approach has been intensively developed and applied to a wide spectrum of system types, see for example [8], [12][22] and the references therein. The ability to globally stabilize the system, and inherent insensitivity to a class of disturbance signals are core properties of the theory. The design of a SMC involves two components. The first prescribes the design of a sliding surface to ensure the nominal equivalent motion to be asymptotically stable. The second is concerned with the synthesis of the control law that drives the trajectory to the sliding surface in finite time and ensures it remains on it thereafter [8]. The surface parameters govern the behavior of the closed loop system. Hence the surface must be designed to ensure the system to exhibit desired dynamics in the sliding mode. Coupled slosh-vehicle dynamics represents a class of underactuated systems. Control of such systems via sliding mode approaches have been investigated by many researchers, (see for example [23] - [27] and the references therein). Wang et al. in [23], considered linear surfaces for the two subsystems corresponding to the actuated subsystem and unactuated subsystem. A second level surface is further designed as a linear combination of the two first level surfaces to synthesize the control so as to ensure the total control contains the equivalent control of both subsystems. The same concept is presented in [24] along with a prediction approach to design optimal surface. Stability analysis of this hierarchical sliding mode approach was discussed by B.L. Ma.[25]. In [26] the sliding surface is designed by considering virtually a fully actuated system and the control is synthesized to ensure the desired switching control in the two subsystems. The design method is simple but the control is complex. Xu et al. proposed a SMC approach to stabilize the indirectly controlled mode of a class of underactuated systems which is in cascade form in [27]. These methods consider the linear sliding surface. This paper proposes a nonlinear sliding surface for improving the damping in an unactuated subsystem for a class of systems.

\section{A. Motivation :}

Coupled vehicle-slosh dynamics constitutes a nonlinear underactuated system. This nonlinear system can be represented by linear system with bounded uncertainty. The corresponding uncertain system representing the coupled slosh-vehicle dynamics contains unmatched uncertainty. Traditional methods are available to design the sliding surface for uncertain systems in the presence of matched uncertainties [8]. However, for mismatched uncertain system very few results are available, see for example [28] and [29]. Ackerman and Özguner [30] considered a back-stepping technique to deal with the unmatched uncertainties while designing a controller for the slosh problem. In [31] a constrained sliding mode control methodology has been developed. A set of initial conditions from which the system can be operated without exceeding a prescribed liquid level are included in a set of constraints. The control challenge is to design a robust sliding surface leading to a simple control structure. The method proposed in
[32]- [34] uses a linear sliding surface. A linear sliding surface calls for a tradeoff between the performance parameters. In this paper, a nonlinear sliding surface is proposed which can offer better closed loop performance. Preliminary results and illustration of the concept was presented in [35], and here full experimental validation and theoretical analysis is included. Also a new proof for the stability of the sliding motion is proposed here. To implement the controller, all the states are required. However, slosh states are difficult to measure. Although various sensors have been reported in the literature, their mounting, accuracy and associated cost make their use questionable. Robust slosh state estimation is therefore required.

\section{B. Outline of Paper:}

The brief outline of the paper is as follows:

In section 2, the system dynamics along with the problem statement is described. Section 3 presents the main results which includes design of the hyperplane, controller synthesis and review of observer development for slosh state estimation. Simulation results are presented in Section 4. Experimental results are presented in Section 5. Finally Section 6 concludes the research findings.

\section{PRoblem Description}

The control objective is to move a partially filled liquid container along a straight line with minimum slosh by applying a control force in the lateral direction. A fundamental mode of the lateral sloshing phenomenon in a moving container is considered. Although Navier-Stokes equations (governing fundamental fluid dynamics) can accurately analyze the dynamic behavior of a liquid, they are extremely complex especially for viscous fluids with a free surface boundary condition [36]. Therefore consideration of such methods is not practicable. A very extensive review of modeling liquid sloshing dynamics can be found in [36]. It describes development of equivalent mechanical models for slosh dynamics. One of the most popular equivalent model approaches uses a pendulum model in which forces and moments generated by the pendulum are matched with those generated by an ideal inviscid fluid. We consider this approach with additional damping for control purposes. The model parameters used are obtained experimentally and verified against this approach [37]. Theoretically a complete mechanical analogy for lateral sloshing demands an infinite number of pendulae, one for each of an infinite number of sloshing modes. It is found that size of each of these masses decreases rapidly with the increase in the mode number [38]. Therefore, it is generally accepted to include only one mass corresponding to a fundamental mode. Here a simple pendulum is considered to represent the sloshing liquid. The remainder of the liquid mass and mass of the tank together form rigid mass. The system is therefore viewed as a moving rigid mass coupled with a simple pendulum moving with respect to fixed $x-z$ reference frame as shown in Fig. 1 . The system parameters are $M_{r}$ : Total rigid mass including mass of the tank, mass of the base plate and mass of liquid that does not slosh. (kg), 


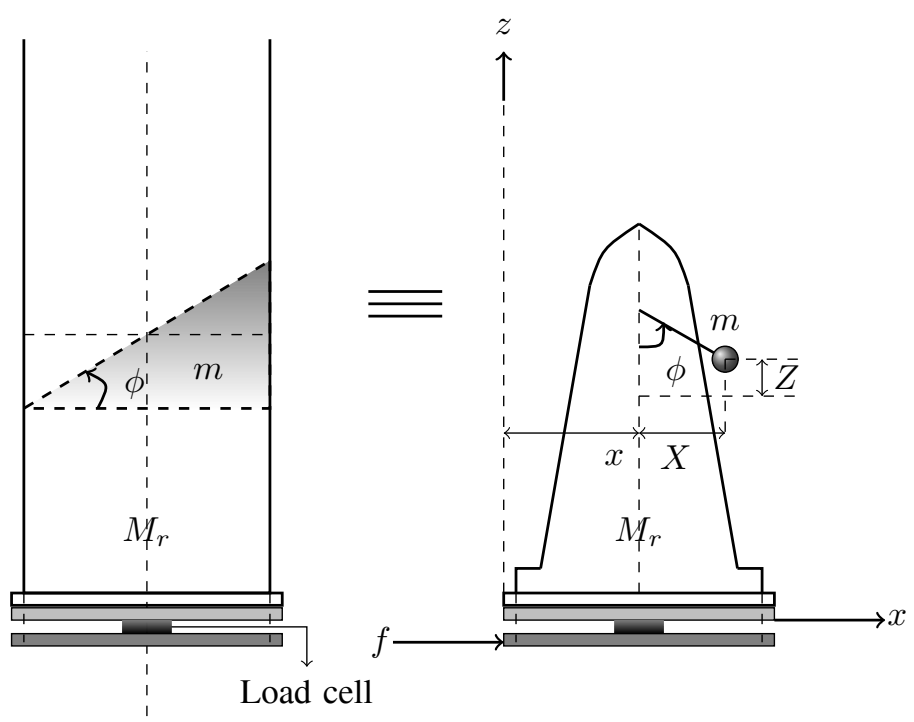

Fig. 1. Representation of slosh by a simple pendulum

$m$ : mass of pendulum (slosh mass) $(\mathrm{kg})$,

$M:$ total mass $\left(M_{r}+m\right)(\mathrm{kg})$,

$M_{l}$ : total mass of the liquid $(\mathrm{kg})$,

$l$ : length of pendulum (meters),

$f$ : force applied for translational motion (Newton),

$x$ : displacement of rigid mass (meters) with reference to fixed reference $x-z$ frame,

$X$ : displacement of $m$ in horizontal direction (meters) with reference to fixed reference $x-z$ frame,

$Z$ : displacement of $m$ in vertical direction (meters),

$\phi:$ pendulum angle or slosh angle (radians),

$c$ : damping coefficient, $\left(\mathrm{kg} \mathrm{m}^{2} / \mathrm{sec}\right)$.

The tank is assumed to be rigid. The liquid is assumed incompressible. Referring to Fig.1.

$$
X=l \sin \phi+x \text { and } Z=l-l \cos \phi .
$$

The kinetic energy of the system is

$$
T=\frac{1}{2} M(\dot{x})^{2}+\frac{1}{2} m(\dot{X})^{2}+\frac{1}{2} m(\dot{Z})^{2} .
$$

The potential energy is, $V=-m g l \cos \phi$.

The Lagrangian of the system is $L=T-V$. The EulerLagrange formulation is

$$
\frac{d}{d t}\left(\frac{\partial L}{\partial \dot{\mathbf{q}}}\right)-\frac{\partial L}{\partial \mathbf{q}}=\mathbf{f}+D(\dot{\mathbf{q}})
$$

where $\mathbf{q}=\left[q_{1}, q_{2}, . .\right]^{T}$ represents generalized variables, one for each degree of freedom (DOF). The term $\mathbf{f}=\left[f_{1}, f_{2} \ldots\right]^{T}$ denotes externally applied forces. $D(\dot{\mathbf{q}})$ represents Rayleigh's dissipation function. For the above system $\mathbf{q}=\left[\begin{array}{ll}x & \phi\end{array}\right]^{T}$ and $\mathbf{f}=\left[\begin{array}{ll}f & 0\end{array}\right]^{T}$. The dynamic equations derived using the EulerLagrange formulation are given below.

$$
\begin{aligned}
M \ddot{x}+m l \cos \phi \ddot{\phi}-m l \dot{\phi}^{2} \sin \phi & =f \\
m l \cos \phi \ddot{x}+m l^{2} \ddot{\phi}+c \dot{\phi}+m g l \sin \phi & =0 .
\end{aligned}
$$

These are nonlinear complex coupled equations. Feedback linearization transforms the stabilization problem of nonlinear system to a stabilization problem of a linear system and hence simplifies the design and analysis. If the nonlinear system has the structure $\dot{\mathbf{x}}=A \mathbf{x}+B \gamma(\mathbf{x})[u-\alpha(\mathbf{x})]$, where $A \in \Re^{n \times n}$, $B \in \Re^{n \times q},(A, B)$ is controllable, the function $\alpha: \Re^{n} \rightarrow \Re^{q}$, $\gamma: \Re^{n} \rightarrow \Re^{q \times q}$, and $\beta(\mathbf{x})=\gamma^{-1}(\mathbf{x})$, then the system can be fully linearized [39], via a state feedback of the form $u=\alpha(x)+\beta(x) v$. Since the given system does not obey the aforementioned condition, it can be partially linearized. Considering

$$
\ddot{x}=u \text {. }
$$

Partially linearized system can be written as below [34].

$$
\begin{aligned}
{\left[\begin{array}{c}
\dot{x} \\
\ddot{x} \\
\dot{\phi} \\
\ddot{\phi}
\end{array}\right] } & =\left[\begin{array}{cccc}
0 & 1 & 0 & 0 \\
0 & 0 & 0 & 0 \\
0 & 0 & 0 & 1 \\
0 & 0 & -g \xi / l & -c / m l^{2}
\end{array}\right]\left[\begin{array}{l}
x \\
\dot{x} \\
\phi \\
\dot{\phi}
\end{array}\right] \\
& +\left[\begin{array}{llll}
0 & 1 & 0 & -(\cos \phi) / l
\end{array}\right]^{T} u,
\end{aligned}
$$

where $\xi=\frac{\sin \phi}{\phi}$.

The system considered is a 2 DOF slosh rig [40]. As described in [33] and [26] (3) can be written as

$$
\dot{\mathbf{x}}=A \mathbf{x}+\Delta A \mathbf{x}+\mathbf{b} u+\Delta \mathbf{b} u,
$$

The problem is to design a sliding mode control for the system (4) to meet the control objective.

\section{MAIN RESULTS}

In (4), $\Delta A, \Delta \mathbf{b}$ are considered as parametric uncertainties. These uncertainties are unmatched uncertainties i.e. they are not implicit in the input channel.

\section{A. Design of hyperplane and controller synthesis}

Consider the sliding surface as given below

$$
s=\mathbf{c}^{T} \mathbf{x}_{e}=0,
$$

where $\mathbf{c}^{T} \in \Re^{1 \times 4}$ and $\mathbf{x}_{e}=\left[\begin{array}{llll}e_{x} & \dot{e_{x}} & e_{\phi} & \dot{e_{\phi}}\end{array}\right]^{T}$ is the error state vector. Define $\mathbf{x}_{d}$ as the desired state vector so that

$$
\mathbf{x}_{e}=\mathbf{x}-\mathbf{x}_{d}
$$

Since the slosh states are to be regulated $\mathbf{x}_{d}=\left[\begin{array}{llll}x_{d} & \dot{x_{d}} & 0 & 0\end{array}\right]^{T}$. Therefore from (4), for a linear or step trajectory the following is valid.

$$
\dot{\mathbf{x}_{d}}=A \mathbf{x}_{d}
$$

A nonlinear sliding surface is proposed which has the following features:

- Unmatched uncertainties belong to the null space of the sliding surface matrix.

- The damping injected in the slosh dynamics is nonlinear and state dependent.

- $c^{T} b=I$.

The proposed sliding surface matrix is

$$
\mathbf{c}^{T}=\left[\begin{array}{llll}
c_{1} & 1 & c_{3} \frac{\sin \phi}{\phi} & 0
\end{array}\right] .
$$

The sliding surface is

$$
s=c_{1} e_{x}+\dot{e_{x}}+c_{3} \sin \phi=0,
$$


This is a nonlinear state dependent surface. Differentiating

$$
\dot{s}=c_{1} \dot{e_{x}}+\ddot{e_{x}}+c_{3} \dot{\phi} \cos \phi
$$

Eqn. (10) can be rewritten as

$$
\dot{s}=\left[\begin{array}{llll}
c_{1} & 1 & c_{3} \cos \phi & 0
\end{array}\right] \dot{\mathbf{x}}_{e}
$$

Gao's power rate reaching law [15] is used.

$$
\dot{s}=-k|s|^{\alpha} \operatorname{sign}(s),
$$

In (12), $k$ and $\alpha$ are tuning parameters used to ensure sliding and minimize the time taken to reach the sliding mode. From (6), (11) and (12)

$$
\left[\begin{array}{llll}
c_{1} & 1 & c_{3} \cos \phi & 0
\end{array}\right]\left(\dot{\mathbf{x}}-\dot{\mathbf{x}_{d}}\right)=-k|s|^{\alpha} \operatorname{sign}(s) .
$$

Substituting (4) and (6)

$$
\begin{array}{r}
{\left[\begin{array}{llll}
c_{1} & 1 & c_{3} \cos \phi & 0
\end{array}\right]\left(A \mathbf{x}_{e}+\mathbf{b} u+\Delta A \mathbf{x}+\Delta \mathbf{b} u\right)} \\
=-k|s|^{\alpha} \operatorname{sign}(s) .
\end{array}
$$

Note that $c^{T} \Delta A=0$ and $c^{T} \Delta b=0$. Therefore the aforementioned equation simplifies to give the control law

$$
u=-k|s|^{\alpha} \operatorname{sign}(s)-c_{1} \dot{e_{x}}-c_{3} \dot{\phi} \cos \phi .
$$

Remark 1: Since the control is synthesized from (12), the existence of the sliding is guaranteed.

Remark 2: The control law in (13) ensures finite time reaching to the sliding surface for the system (4). The surface is so designed that it cancels the effects of unmatched uncertainties. The switching gain is chosen to satisfy reaching condition.

The nonlinearities in the partial feedback linearized system can be canceled if it is known exactly. In this situation the control yields sliding modes if $k>\delta$, where $\delta$ is an arbitrary positive constant. However under parametric variations the nonlinearities to be canceled are not known exactly. Hence it can not be fully canceled by partial feedback linearization technique. These nonlinearities can be considered as uncertainties which are matched and bounded. To guarantee the existence of sliding modes in presence of matched uncertainties the switching gain of SMC must be greater than the maximum bound of the uncertainty i.e. $\rho_{\max }$. This satisfies the $\eta$ reachability condition and hence ensures the existence of sliding modes. It may be noted that the unmatched uncertainties in (4). are taken care by the design of the sliding surface. Eqn. (1a) can be written as

$$
\ddot{x}=\frac{f}{M}+\Delta_{n l},
$$

where $\Delta_{n l}$ is the nonlinear term which includes a quadratic term. Considering the bounds of $\phi$ and the values of slosh parameters,

$$
\begin{aligned}
\frac{m g \sin 2 \phi}{2 M} & \in(-1.1,1.1), \\
\frac{c \dot{\phi} \cos \phi}{M l} & \in 9.7537 e-004 \dot{\phi}(0,1) \approx 0: \text { neglected }, \\
\frac{m l \dot{\phi}^{2} \sin \phi}{M} & \in(-0.0115,0.0115) \dot{\phi}^{2}, \\
\left|\Delta_{\text {nlmax }}\right| & =3.7 .
\end{aligned}
$$

Further consideration of $\pm 20 \%$ parametric uncertainties gives

$$
\left|\Delta_{n l \max }\right| \equiv \rho_{\max }=5.55 \text {. }
$$

The bounds on $\dot{\phi}$ were computed by considering maximum slosh amplitude $(\pi / 2)$ and maximum slosh frequency (close to resonant frequency $2.0 \mathrm{~Hz}$ ) for the first mode in simulation which is $15 \mathrm{rad} / \mathrm{sec}$.

It may be noted that $\Delta_{n l}$ lies in the input channel and hence constitutes matched lumped disturbance. As is proved in the $\eta$ reachability condition [8], finite time reaching is assured if the gain of the discontinuous control i.e. $k>\rho_{\max }$. Hence by proper choice of gain $k$ a finite time reaching is guaranteed which means finite time escape is avoided.

It is a well known fact that low damping results in an increase in speed of response, associated with large overshoot. Increase in damping results in reduction in overshoot. In order to get high speed of response together with low overshoot it is essential to have low damping initially and increase it subsequently as slosh angle decreases. From equation (18), the slosh dynamics during sliding are

$$
\begin{aligned}
\ddot{\phi}= & -20 c_{1}^{2} \cos \phi e_{x}-\left(188.2 \xi+20 c_{1} c_{3} \cos \phi\right) \phi \\
& +\left(20 c_{3}(\cos \phi)^{2}-0.085\right) \dot{\phi} .
\end{aligned}
$$

This is a second order slosh dynamics. Due to the assumption of bounded $\phi$, the characteristic polynomial describing the above slosh dynamics is an interval polynomial. The term $\left(20 c_{3}(\cos \phi)^{2}-0.085\right)$ contributes damping which varies nonlinearly.

Remark 3: The damping injected in the slosh dynamics varies nonlinearly. For a large value of $\phi$, damping is less and this enhances the speed of response. As $\phi$ decreases, damping increases which reduces the overshoot. The proposed nonlinear sliding surface thus enhances the performance of the slosh dynamics.

\section{B. Stability Analysis}

From (3), (6) and (7),

$$
\begin{aligned}
\dot{\mathbf{x}}_{e} & =\left[\begin{array}{cccc}
0 & 1 & 0 & 0 \\
0 & 0 & 0 & 0 \\
0 & 0 & 0 & 1 \\
0 & 0 & -g \xi / l & -c / m l^{2}
\end{array}\right] \mathbf{x}_{e} \\
& +\left[\begin{array}{llll}
0 & 1 & 0 & -(\cos \phi) / l
\end{array}\right]^{T} u,
\end{aligned}
$$

where $\mathbf{x}_{e}=\left[\begin{array}{llll}e_{x} & \dot{e_{x}} & \phi & \dot{\phi}\end{array}\right]^{T}$ is the error state vector. Using the transformation $\mathbf{z}=T_{r} \mathbf{x}_{e}$ to get

$$
\begin{aligned}
& \mathbf{z}=\left[\begin{array}{llll}
e_{x} & \phi & \dot{\phi} & \dot{e_{x}}
\end{array}\right]^{T} \equiv\left[\begin{array}{ll}
\mathbf{z}_{1} & \mathbf{z}_{2}
\end{array}\right]^{T}, \\
& \mathbf{z}_{1} \in \Re^{3} \text { and } \mathbf{z}_{2} \in \Re^{1} \text {. }
\end{aligned}
$$

Therefore (14) in $\mathbf{z}$ coordinates can be written as

$$
\left[\begin{array}{c}
\dot{\mathbf{z}_{1}} \\
\dot{\mathbf{z}_{2}}
\end{array}\right]=\left[\begin{array}{ll}
A_{11} & A_{12} \\
A_{21} & A_{22}
\end{array}\right]\left[\begin{array}{l}
\mathbf{z}_{1} \\
\mathbf{z}_{2}
\end{array}\right]+\left[\begin{array}{l}
b_{1} \\
b_{2}
\end{array}\right] u,
$$


where

$$
\begin{array}{ccc}
A_{11}= & {\left[\begin{array}{ccc}
0 & 0 & 0 \\
0 & 0 & 1 \\
0 & -g \xi / l & -c / m l^{2}
\end{array}\right],} & A_{12}=\left[\begin{array}{l}
1 \\
0 \\
0
\end{array}\right], \\
A_{21}=\left[\begin{array}{ccc}
0 & 0 & 0
\end{array}\right], & A_{22}=0, \\
b 1=\left[\begin{array}{lll}
0 & 0 & -\frac{\cos \phi}{l}
\end{array}\right]^{T}, & b_{2}=1 .
\end{array}
$$

The sliding variable in $\mathbf{z}$ coordinates is

$$
s=\left[\begin{array}{llll}
c_{1} & c_{3} \frac{\sin \phi}{\phi} & 0 & 1
\end{array}\right] \mathbf{z}=0 . \Rightarrow s=s_{1} \mathbf{z}_{1}+s_{2} \mathbf{z}_{2}=0,
$$

where $s_{1} \in \Re^{1 \times 3}$ and $s_{2} \in \Re^{1 \times 1}$. The reduced order dynamics during sliding is obtained by substituting $\mathbf{z}_{2}=-s_{1} \mathbf{z}_{1}$ as

$$
\dot{\mathbf{z}}_{1}=\left[\begin{array}{ccc}
0 & 0 & 0 \\
0 & 0 & 1 \\
0 & -g \xi / l & -c / m l^{2}
\end{array}\right] \mathbf{z}_{1}-\left[\begin{array}{l}
1 \\
0 \\
0
\end{array}\right] s_{1} \mathbf{z}_{1}+\mathbf{b}_{1} u_{e q}
$$

The equivalent control $u_{e q}$ is obtained from $\dot{s}=0$.

$$
u_{e q}=-c_{1} \dot{e_{x}}-c_{3} \cos \phi \dot{\phi}
$$

From the equation of the surface, $\dot{e}_{x}=-c_{1} e_{x}-c_{3} \sin \phi$. Therefore $u_{e q}$ can be written as below.

$$
u_{e q}=c_{1}^{2} e_{x}+c_{1} c_{3} \xi \phi-c_{3} \dot{\phi} \cos \phi
$$

Substituting $s_{1}$ and $u_{e q}$ in (15). to get equations governing the dynamics of the sliding motion as below:

$$
\begin{gathered}
\dot{e}_{x}=-c_{1} e_{x}-c_{3} \sin \phi \\
\ddot{\phi}=-\frac{c_{1}^{2} \cos \phi}{l} e_{x}-\left(\frac{g}{l}+\frac{c_{1} c_{3} \cos \phi}{l}\right) \sin \phi \\
+\left(-\frac{c}{m l^{2}}+\frac{c_{3}(\cos \phi)^{2}}{l}\right) \dot{\phi}
\end{gathered}
$$

The Eqns. (16) and (17) can be rewritten as below.

$$
\begin{aligned}
\dot{e_{x}} & =-c_{1} e_{x}-c_{3} \sin \phi \\
\dot{\phi} & =-\phi+\phi+\dot{\phi} \\
\ddot{\phi} & =-h_{1} e_{x}-h_{2} \phi+h_{3} \dot{\phi}
\end{aligned}
$$

where,

$$
\begin{aligned}
h_{1} & =\frac{c_{1}^{2}}{l} \cos \phi \\
h_{2} & =\left(\frac{g}{l}+\frac{c_{1} c_{3} \cos \phi}{l}\right) \frac{\sin \phi}{\phi} \\
h_{3} & =-\frac{c}{m l^{2}}+\frac{c_{3} \cos ^{2} \phi}{l}=-0.09+\frac{c_{3} \cos ^{2} \phi}{l} .
\end{aligned}
$$

Define $p=\phi+\dot{\phi}$, therefore $\dot{p}=\ddot{\phi}+\dot{\phi}$. The above equations (18), (19) and (20) can be written as below

$$
\begin{aligned}
\dot{e_{x}} & =-c_{1} e_{x}-c_{3} \sin \phi \\
\dot{\phi} & =-\phi+p \\
\dot{p} & =\left(1+h_{3}\right) p-h_{1} e_{x}-h_{2} \phi-\left(1+h_{3}\right) \phi .
\end{aligned}
$$

These equations represent interconnected system of the form

$$
\begin{aligned}
\dot{e_{x}} & =f_{1}\left(e_{x}\right)+g_{1}(\phi) \\
\dot{\phi} & =f_{2}(\phi)+g_{2}(p) \\
\dot{p} & =f_{3}(p)+g_{3}\left(e_{x}, \phi\right) .
\end{aligned}
$$

The interconnected system is decomposed into isolated subsystems ignoring the interconnections. The stability of each subsystem is analyzed. By combining these results together with the information of the interconnections, the conclusions about the stability of the interconnected system can be drawn [39].

We derive the conditions to ensure each of the isolated subsystems is stable. Further a composite Lyapunov function is considered to analyze the stability of the total interconnected system. Considering the first isolated system neglecting the interconnections

$$
\dot{e_{x}}=-c_{1} e_{x}
$$

Consider a Lyapunov function candidate

$$
V_{1}=0.5 e_{x}^{2}
$$

Differentiating $V_{1}$

$$
\dot{V_{1}}=e_{x} \dot{e_{x}} .
$$

Substituting for $\dot{e_{x}}$ to get

$$
\dot{V}_{1}=-c_{1} e_{x}^{2}<0 .
$$

The aforementioned equation implies that the first isolated subsystem is stable if $c_{1}>0$.

The second isolated subsystem is

$$
\dot{\phi}=-\phi \text {. }
$$

Consider a candidate Lyapunov function

$$
V_{2}=0.5 \phi^{2} .
$$

Differentiating to get

$$
\dot{V}_{2}=-\phi^{2} .
$$

This subsystem is stable. The third isolated subsystem is

$$
\dot{p}=\left(1+h_{3}\right) p .
$$

Consider a candidate Lyapunov function

$$
V_{3}=0.5 p^{2} \text {. }
$$

Differentiating to get

$$
\dot{V}_{3}=p \dot{p}=\left(1+h_{3}\right) p^{2} .
$$

For this system to be stable, $\left(1+h_{3}\right)<0$

$$
\text { i.e. } 1+\left(-0.09+\frac{c_{3} \cos ^{2} \phi}{l}\right)<0 .
$$

With $c_{3}<0$ the system is stable if

$$
\begin{aligned}
0.91-\frac{\left|c_{3}\right| \cos ^{2} \phi}{l} & <0 \\
\Rightarrow \cos ^{2} \phi & >\frac{0.91}{20\left|c_{3}\right|}
\end{aligned}
$$

For the chosen $c_{3}=-1$, the range of $\phi$ for which the isolated system is stable, is $-77.6^{\circ} \leq \phi \leq 77.6^{\circ}$. This range can be increased by choosing $c_{3}$ more negative. It may be noted that for practical systems the lateral slosh angle is such that $-75^{\circ} \leq \phi \leq 75^{\circ}$. Thus choice of $c_{1}>0$ and $c_{3}=-1$ assures the stability of all the isolated subsystems. 
Now to analyze the stability of the composite interconnected system, a composite Lyapunov function is chosen as below.

$$
V=V_{1}+V_{2}+V_{3}+\frac{g(1-\cos \phi)}{l}-\frac{\left|c_{1} c_{3}\right|(1-\cos 2 \phi)}{4 l} .
$$

Substituting for $V_{1}, V_{2}, V_{3}$ in the above equation

$V=0.5 e_{x}^{2}+0.5 \phi^{2}+0.5 p^{2}+\frac{g(1-\cos \phi)}{l}-\frac{\left|c_{1} c_{3}\right|(1-\cos 2 \phi)}{4 l}$

For $V$ in (35) to be positive definite

$$
\frac{g(1-\cos \phi)}{l}-\frac{\left|c_{1} c_{3}\right|(1-\cos 2 \phi)}{4 l}>0 .
$$

From the above $V$ is positive definite if

$$
\left|c_{1} c_{3}\right|<g \text {. }
$$

Also note that selection of the sliding surface parameters that satisfy the above conditions i.e. $c_{1}>0, c_{3} \leq-1$ and $\left|c_{1} c_{3}\right|<$ $g$ yield

$$
\left.\begin{array}{cc}
h_{1} & >0 \\
h_{2} & >0 \\
\left(1+h_{3}\right) & <0
\end{array}\right\}
$$

Differentiating $V$ to get

$$
\dot{V}=e_{x} \dot{e_{x}}+\phi \dot{\phi}+p \dot{p}+\left(\frac{g}{l}-\frac{\left|c_{1} c_{3}\right| \cos \phi}{l}\right) \dot{\phi} \sin \phi .
$$

Using (22) and $c_{3} \leq-1$,

$$
\dot{V}=e_{x} \dot{e_{x}}+\phi \dot{\phi}+p \dot{p}+h_{2} \phi \dot{\phi}
$$

Using (24), (25) and (26) with the information of $c_{3}<0$ and $\dot{\phi}=p-\phi$,

$$
\begin{aligned}
\dot{V}= & e_{x}\left(-c_{1} e_{x}+\left|c_{3}\right| \sin \phi\right)+\phi(p-\phi) \\
& +p\left(1+h_{3}\right) p-p h_{2} \phi-p h_{1} e_{x}-p\left(1+h_{3}\right) \phi \\
& +p h_{2} \phi-h_{2} \phi^{2} .
\end{aligned}
$$

Defining $\left|c_{3}\right| \frac{\sin \phi}{\phi}=h_{4}$ and simplifying the above to

$$
\begin{aligned}
\dot{V}= & -c_{1} e_{x}^{2}+h_{4} e_{x} \phi-\phi^{2}\left(1+h_{2}\right) \\
& +p^{2}\left(1+h_{3}\right)-p h_{1} e_{x}-p h_{3} \phi .
\end{aligned}
$$

Note that $h_{4}>0$. Using (37) the above can be simplified to

$$
\begin{aligned}
\dot{V}= & -c_{1} e_{x}^{2}+h_{4} e_{x} \phi-\phi^{2}\left(1+h_{2}\right) \\
& -p^{2}\left|\left(1+h_{3}\right)\right|-p h_{1} e_{x}+p\left|h_{3}\right| \phi . \\
\Rightarrow \dot{V} & =-\left[\begin{array}{lll}
e_{x} & \phi & p
\end{array}\right] Q\left[\begin{array}{c}
e_{x} \\
\phi \\
p
\end{array}\right] .
\end{aligned}
$$

where

$$
Q=\left(\begin{array}{ccc}
c_{1} & -h_{4} & 0 \\
0 & 1+h_{2} & 0 \\
h_{1} & -\left|h_{3}\right| & \left|\left(1+h_{3}\right)\right|
\end{array}\right)
$$

It is obvious from (38)that the composite system is stable if $Q$ is positive definite. For $\mathrm{Q}$ to be positive definite the following must hold

$$
\begin{aligned}
c_{1} & >0 \\
c_{1}\left(1+h_{2}\right) & >0 \\
c_{1}\left(1+h_{2}\right)\left|\left(1+h_{3}\right)\right| & >0 .
\end{aligned}
$$

For the chosen surface parameters $c_{1}=1$ and $c_{3}=-1$, all the above conditions are satisfied. Hence with the positive definite matrix, the total interconnected system is asymptotically stable.

Remark 4: The given interconnected system is stable if the sliding surface parameters are such that $c_{1}>0, c_{3}<0$, $\left|c_{1} c_{3}\right|<g$.

The approach is based on partial feedback linearization which requires perfect knowledge of the plant parameters for canceling the effect via feedback. Nonlinearities which include nominal parameters are compensated by feedback. However, further variation of parameters and nonlinearities can be considered as uncertainties contributing matched uncertainties and hence are compensated by SMC. Equation (1a) can be written as

$$
f=M u+\Delta_{n l} \pm \Delta_{p}
$$

where $\Delta_{n l}=m l \cos \phi D-m l \dot{\phi}^{2} \sin \phi$ are the nonlinearities compensated in the feedback. These include nominal parameters, as $\pm \Delta_{p}$ represents uncertainties due to parameter variations. It should be noted that these are matched. While designing the nonlinear part of the sliding mode control, the maximum bound of $\Delta_{p}$ is considered. One can consider $\Delta_{n l}$ and $\Delta_{p}$ together as lumped uncertainties, but this will increase the total bound to be considered while designing the nonlinear part of SMC.

\section{Design of sliding mode observer}

A sliding mode observer for the slosh state estimation using the high gain approach proposed in [41] and [34], is used here. The system in (1) can be written as below:

$$
\begin{aligned}
\dot{\mathbf{x}_{a}} & =\mathbf{f}_{a}(\mathbf{x})+\mathbf{b}_{a} u, \\
\dot{\mathbf{x}_{r}} & =\mathbf{f}_{r}(\mathbf{x})+\mathbf{b}_{r} u,
\end{aligned}
$$

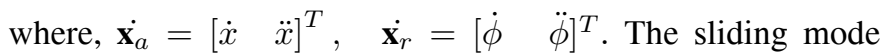
observer dynamics is

$$
\begin{aligned}
\dot{\widehat{\mathbf{x}_{a}}} & =\mathbf{f}_{a}(\widehat{\mathbf{x}})+\mathbf{b}_{a} u+K \mathbf{m} \operatorname{sign}(\mathbf{s}) \\
\dot{\hat{\mathbf{x}_{r}}} & =\mathbf{f}_{r}(\widehat{\mathbf{x}})+\mathbf{b}_{r} u+L_{r} K \mathbf{m} \operatorname{sign}(\mathbf{s}),
\end{aligned}
$$

where $\widehat{\mathbf{x}}$ is a vector of state estimates and $\widehat{\mathbf{x}_{a}}$ is estimate of measurable states. $L_{r} \in \Re^{2 \times 2}$. $K$, and sign (s) both $\in \Re^{2 \times 2}$, $\mathbf{m}=\left[\left|s_{1}\right|^{\alpha}\left|s_{2}\right|^{\alpha}\right]^{T}$ and $0<\alpha<1$. The switching function is defined as

$$
\mathbf{s}=\mathbf{x}_{a}-\widehat{\mathbf{x}_{a}} \equiv \widetilde{\mathbf{x}_{a}} .
$$

Subtracting (40) from (39) gives the observer error dynamics as below.

$$
\begin{aligned}
\dot{\widetilde{\mathbf{x}_{a}}} & =\Delta \mathbf{f}_{a}-K \mathbf{m} \operatorname{sign}(\mathbf{s}), \\
\dot{\tilde{\mathbf{x}}_{r}} & =\Delta \mathbf{f}_{r}-L_{r} K \mathbf{m} \operatorname{sign}(\mathbf{s}),
\end{aligned}
$$

where $\Delta \mathbf{f}_{a} \equiv \mathbf{f}_{a}(\mathbf{x})-\mathbf{f}_{a}(\widehat{\mathbf{x}})$ and $\Delta \mathbf{f}_{r} \equiv \mathbf{f}_{r}(\mathbf{x})-\mathbf{f}_{r}(\widehat{\mathbf{x}})$ $\widetilde{\mathbf{x}_{r}} \equiv \mathbf{x}_{r}-\widehat{\mathbf{x}_{r}} . K$ is a diagonal matrix and is designed to ensure sliding. The values of $k_{1}, k_{2}$ are chosen as $k_{1}=k_{2}=25$. 
During sliding, $\widetilde{\mathbf{x}_{a}}=0$. From (42a) and (42b), the reduced observer error subsystem obtained using the equivalent control approach is

$$
\dot{\widetilde{\mathbf{x}_{r}}}=\Delta \mathbf{f}_{r}-L_{r} \Delta \mathbf{f}_{a} .
$$

Linearizing about $\widetilde{\mathbf{x}_{r}}=0$ to get

$$
\dot{\widetilde{\mathbf{x}_{r}}}=\left[A_{r}-L_{r} C_{r}\right] \widetilde{\mathbf{x}_{r}}+\delta_{r},
$$

The term $\delta_{r}$ represents parametric uncertainties and nonlinearities in the reduced subsystem. It is state dependent and uniformly norm bounded.

$$
A_{r}=\left[\begin{array}{cc}
0 & 1 \\
0 & 0.085
\end{array}\right], \quad C_{r}=\left[\begin{array}{ll}
0 & 0 \\
1 & 0
\end{array}\right] .
$$

The pair $\left(A_{r}, C_{r}\right)$ must be observable. $L_{r}$ is designed to ensure $\left(A_{r}-L_{r} C_{r}\right)$ Hurtwitz. Further, to take care of uncertainties and prescribe faster convergence of the observation error to zero, $L_{r}$ is chosen to be

$$
L_{r}=\left[\begin{array}{ll}
0 & 101.9150 \\
0 & 191.3372
\end{array}\right] .
$$

The closed loop poles of the reduced order observer system are -2 and -100 and stable dynamics are therefore assured. Remark5: It should be noted that the estimation error is like a disturbance which decays. Therefore, by choosing the switching gain $k$ greater than that of the designed value for a state based SMC will ensure finite time reaching to the surface $s$ even with a SMO based SMC.

\section{Simulation Results}

The validity of the proposed scheme was tested in simulation. The states were estimated using the sliding mode observer discussed in the previous section. The controller parameters were $k=6 ; \alpha=0.5 ; \quad \delta=0.005$. A sigmoid function was used to implement the SMC to alleviate chattering. The problem of discontinuous injection is no longer pertinent in the SMO context. Therefore signum function was used in SMO. A step command of $100 \mathrm{~mm}$ was applied at time $t=0.615 \mathrm{sec}$. Fig. 2 shows that the container settles at the desired position in about 4 seconds. The slosh is nicely regulated as shown in Fig.3. The switching function in Fig. 4 illustrates the fast reaching phase. Sliding commences in less that 0.5 seconds. The control in Fig. 5 shows initial overshoot for a very short period when there are step changes in the command signal. For the remainder of the operating time the control signal is smooth.

\section{EXPERIMENTAL RESUlTS}

The proposed controller was implemented on an experimental setup developed for slosh parameter identification.

\section{A. Experimental setup}

The experimental set up is as shown in the Fig.(6). An acrylic cylindrical tank is mounted on a platform. The dimensions of the tank are height $=450 \mathrm{~mm}$ and diameter $=190 \mathrm{~mm}$. Two degrees of actuation, one for translation and the other for pitching are provided using separate permanent

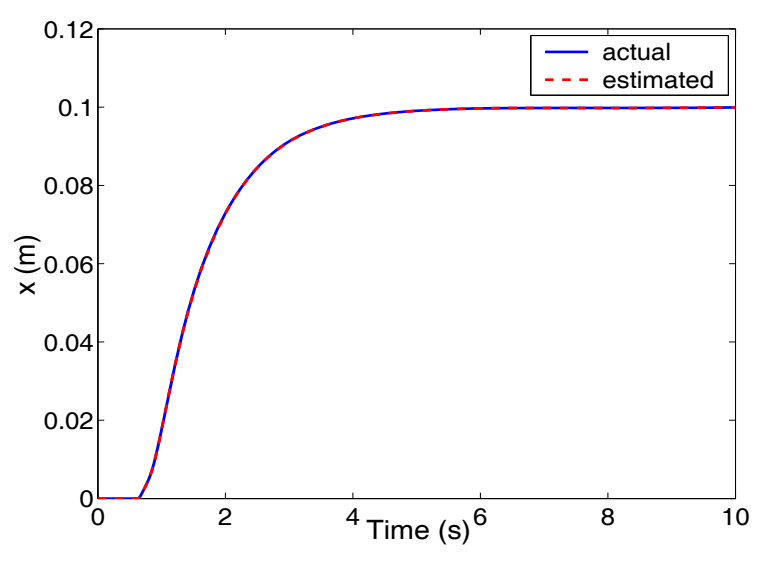

Fig. 2. Container position: simulated results

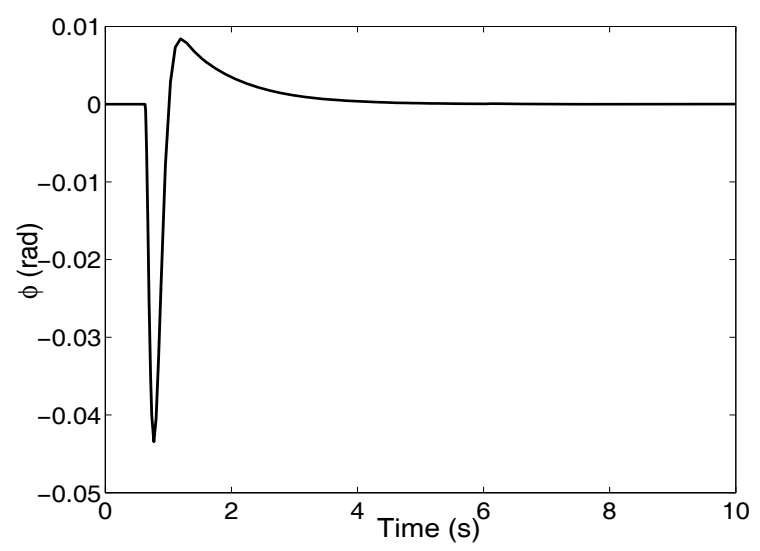

Fig. 3. Slosh position: simulated results

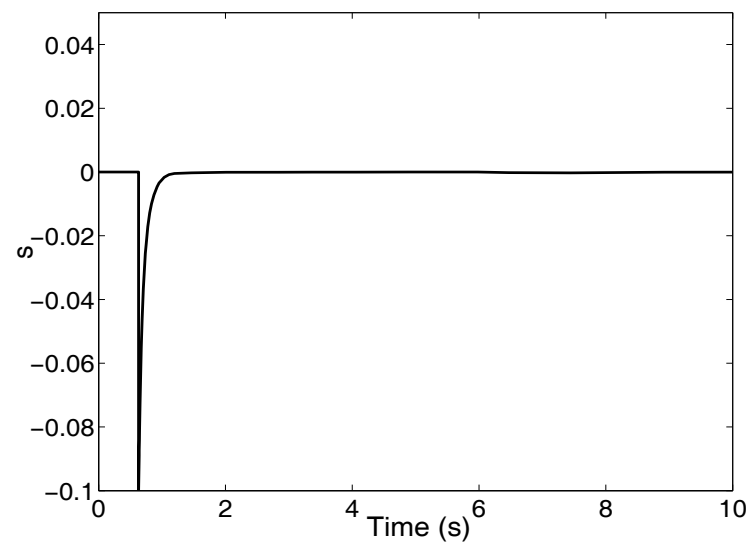

Fig. 4. Switching function: simulated results

magnet brush-less DC servomotors through a ball screw linear slide arrangement. The motion control drives are configured to run these motors in torque control mode from an analog input. An incremental encoders are mounted on the motor shafts to capture position data. This incremental shaft encoder in combination with the DC servomotor is used for indication of both, shaft positioning as well as velocity. The shaft is coupled to ball screw to effect rotary to linear transformation. By considering the pitch factor of the ball screw, measurements of linear position $(x)$ and velocity $(\dot{x})$ are obtained. We make it 


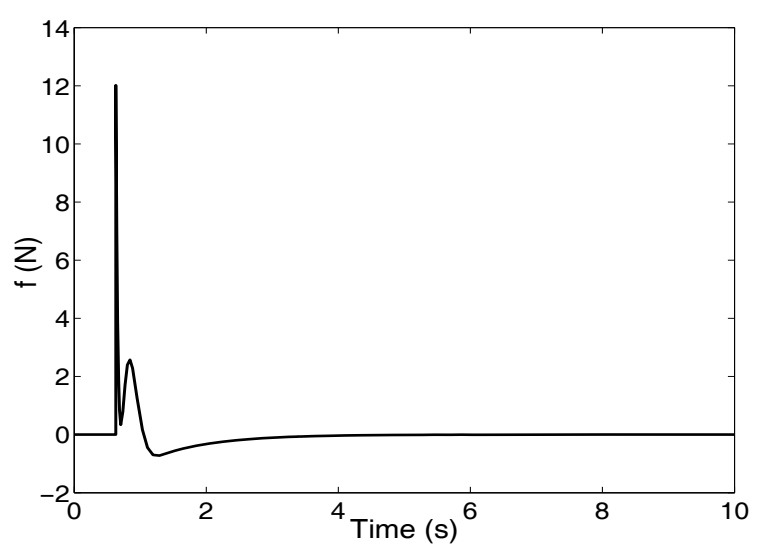

Fig. 5. Control efforts: simulated results

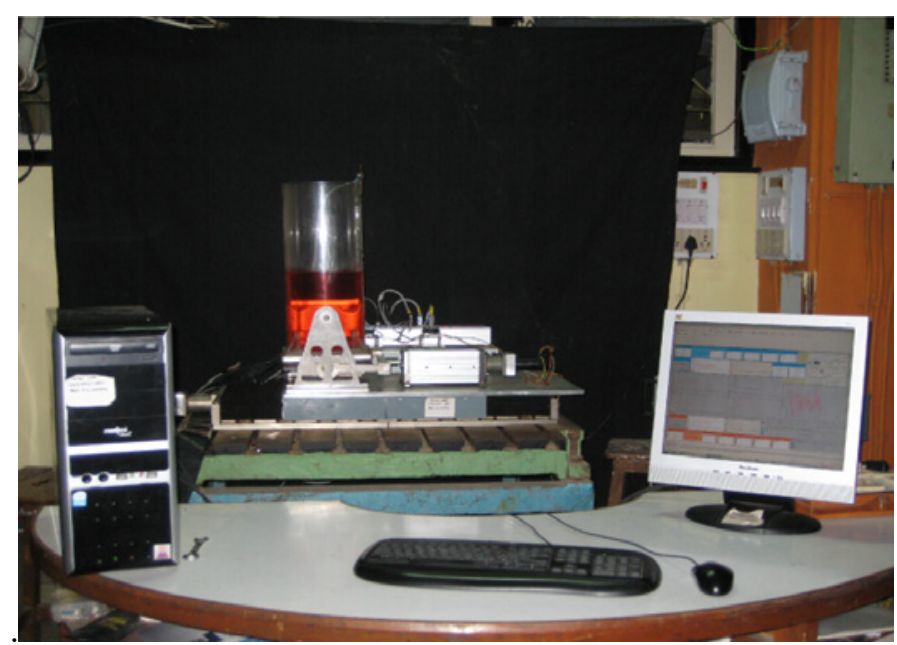

Fig. 6. Moving container System

clear that only one motor was used in the experiments. A six axis load cell is mounted directly beneath the tank to capture force and moment data . A dSPACE ds 1104 card provides the real time interface. Sensor signals are captured and the actuators are controlled using Simulink and the dSPACE interface. A real time interface (RTI) from Matlab enables the simulink control model to link to the micro-controller in the dSPACE card for real time implementation. we have used only one motor (for lateral excitation) for implementing the proposed controller. The sampling period has been chosen as $1 \mathrm{msec}$, which is very small for the mechanical system under consideration. Hence continuous algorithms can be implemented as directly.

\section{B. Implementation Scheme}

The SMC was designed to control slosh in a container using the acceleration of the container as the control. The necessary force required to be applied to the plant is obtained from (14). This force was applied to the plant model directly to study the performance in simulation. In simulation, the PID control was not used as the force to be applied was available for direct implementation in simulation.

A stable PID controller to drive a servo system (drive stage, actuator, ball screw etc.), to follow a reference trajectory accurately (more that $95 \%$ ) for slosh parameter identification has been used. The PID provides position control but not slosh suppression. The SMC law provides the plant acceleration required to meet the desired objectives. The implementation must ensure this acceleration and is achieved by ensuring the plant follows a position reference which ensures this acceleration. From the desired acceleration obtained from the SMC, the necessary position reference for the PID was obtained by integrating the acceleration twice. This is one way to generate the command [42] -[45]. Alteratively one can consider the model with actuator, ball-screw, sensor etc. and redesign the control; the designed SMC would give some control input to be applied directly to the actuator. However, this discontinuous control, theoretically of infinite frequency required to achieve ideal sliding may not be practically applied. Here the PID mechanism has been used for implementing the SMC law in a simple practical way thereby verifying the proposed scheme. The methodology has the added advantage that the position reference obtained from the discontinuous desired acceleration reference is smooth. The schematic for implementation may be referred from [34]. When the actual plant acceleration does not equal to the desired acceleration, the equivalent force $f_{s m}+\Delta f$ is applied to the plant. Note that $\Delta f$ is a matched disturbance hence is taken care of by the switching component to ensure sliding assuming the switching component gain is greater than the maximum value of $\Delta f$. Thus the existence of sliding is ensured. A properly tuned PID mechanism together with sliding mode control to generate the desired reference gives a stable closed-loop performance.

\section{Slosh Estimator Validation}

The slosh state estimator was validated using pressure sensor data, the force sensor data and the images captured from the camera. A force sensor mounted beneath the tank measures forces and moments acting on the tank. Under the lateral sinusoidal excitation, the force measured in the lateral direction shows the trend of the slosh force. The trend of the force sensor data matches closely with the trend of SMO estimates

A pressure sensor was mounted on the top, close to the tank wall. A sinusoidal excitation was applied. The SMO estimate calibrated in terms of liquid level and the pressure sensor measurements calibrated in terms of liquid level were closely matching in steady state. It was observed that pressure sensor measurements were noisy. Use of filter added delay. Further it could not give accurate measurements during initial transient period.

The camera was mounted on the stationary wooden stand. A sinusoidal excitation was applied to the plant. The initial transients as well as the steady state performance were recorded by the camera. The image frames were captured using virtual dub software. The images at certain instants of time were processed off line. The edge detection algorithm yields the instantaneous level of the liquid from the image of the container. The slosh angles calibrated in terms of liquid level were noted at those instants of time during transient as well as steady state and were found in close agreement with the camera data. 


\section{SMC with SMO}

The experimental results of the proposed scheme in Fig.7 to Fig. 10 correlate well with the simulation results. Noise was present in the $\dot{x}$ measurement which corrupted the slosh state estimation. A properly tuned butterworth filter was used to filter this noise. Use of a properly tuned filter has given cleaned estimates. These estimates were used to implement the controller.

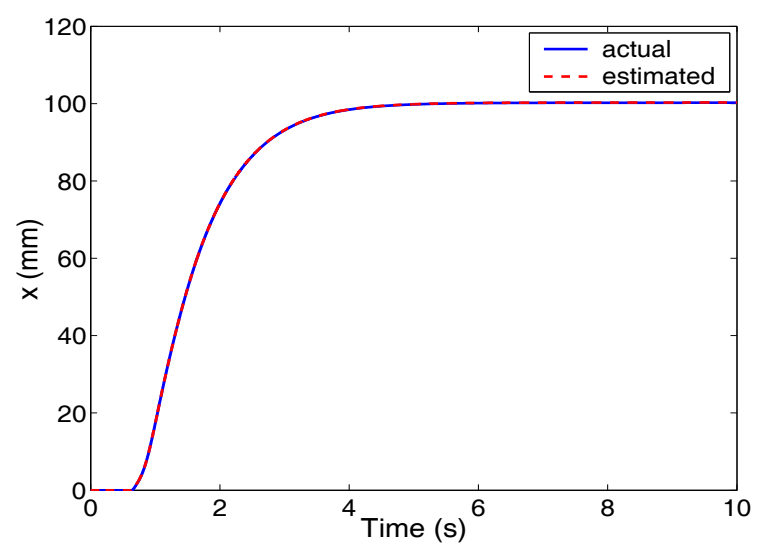

Fig. 7. Container position: experimental results

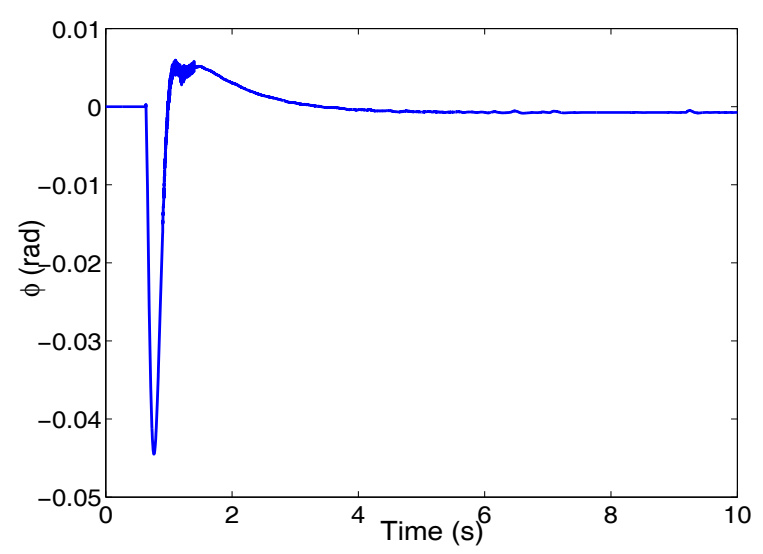

Fig. 8. Slosh position from SMO: experimental results

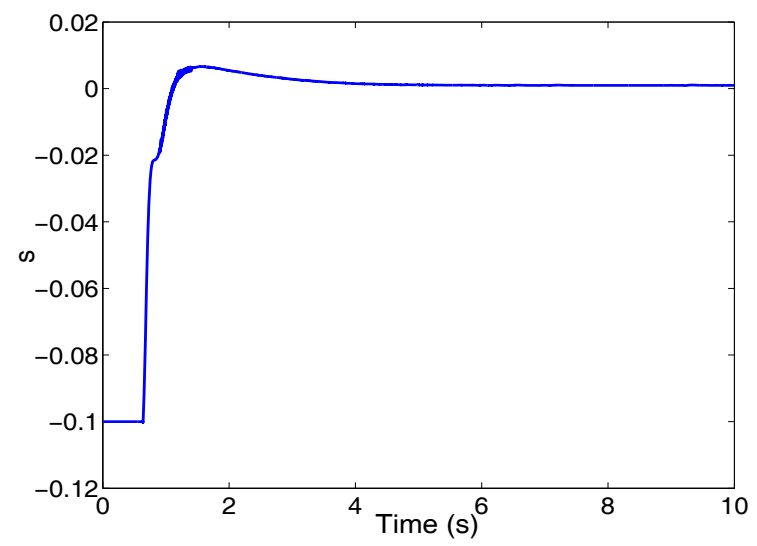

Fig. 9. Switching function: experimental results

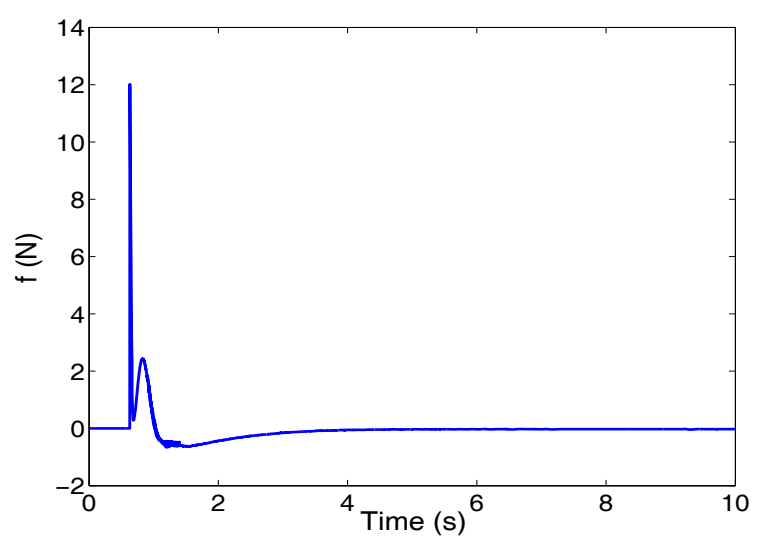

Fig. 10. Control efforts: experimental results

\section{E. PID control}

A step command was applied for the container to move in 4 seconds to $100 \mathrm{~mm}$. The SMC component was switched off. The PID controller gains were tuned to $K_{p}=0.025$, $K_{i}=0.09$ and $K_{d}=0.0022$. Fig. 11 and 12 show that the desired $\mathrm{x}$ dynamics can be achieved but not the desired slosh suppression. Comparison of the performance shown in Fig.8 and Fig.12 illustrates the strength of the SMC.

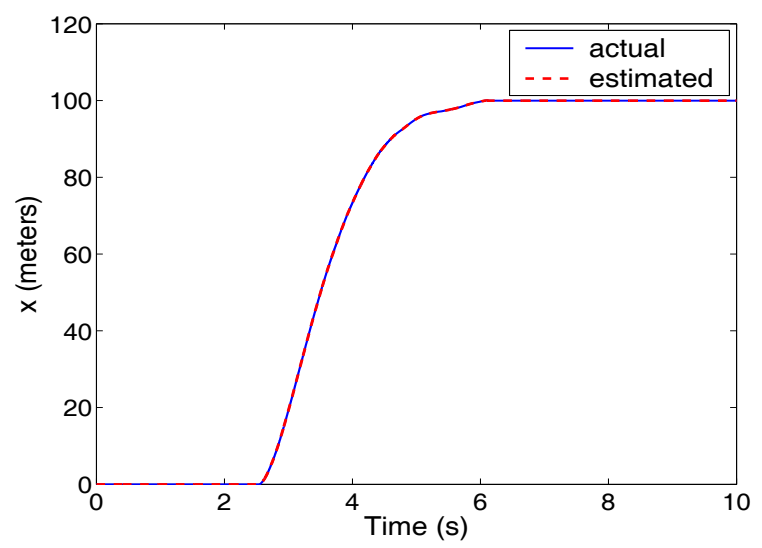

Fig. 11. Experimental Performance of PID controller and SMO

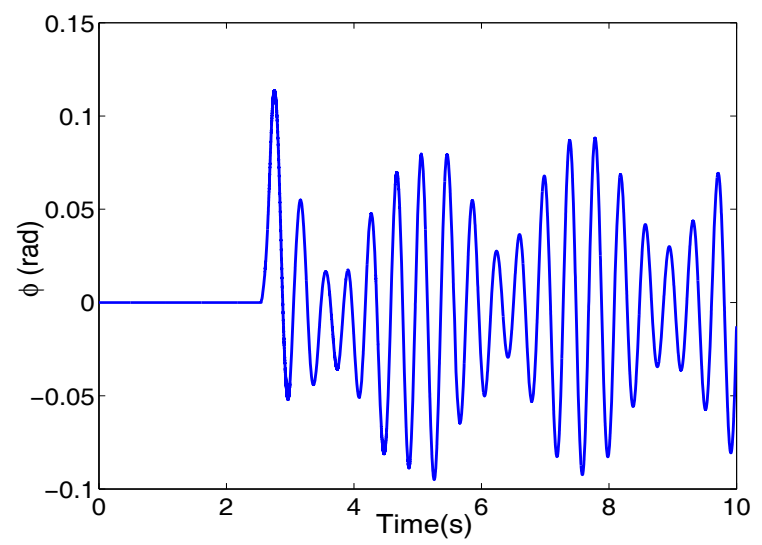

Fig. 12. Experimental Performance of PID controller and SMO 


\section{CONCLUSiOnS}

In this paper, the problem of control of slosh has been considered. The plant dynamics is a second order underactuated system. Partial feedback linearization was used. The partially linearized system was represented as a linear uncertain plant which has uncertainties in both the system and input matrices. A method has been proposed to design a stable surface having the additional feature of enhancing the damping of the slosh dynamics. This surface yields a simple, implementable control law. Slosh states have been estimated using a sliding mode observer. The experimental results closely match with the simulation results.

Noise due to electromagnetic interference was observed. Filters are used to filter the sensor signals. However filters add delays. Sophisticated filters can be used to improve the performance of the controller by minimizing the delays. If the estimation of the uncertainties is also available along with the states then the control will be less conservative. The controller performance can be further improved by adaptive tuning of the controller parameters. The proposed methodology has the potential for application to a wide spectrum of problems.

\section{ACKNOWLEDGEMENT}

The work is partially supported by Royal Academy of Engineering, London when B. Bandyopadhyay was visiting University of Kent as a Distinguished Visiting Fellow of the said Academy.

\section{REFERENCES}

[1] C. Nichkawde, P. Harish, and N. Ananthkrishnan, "Stability analysis of a multibody system model for coupled sloshvehicle dynamics," J. Sound Vib., vol. 275, no. 3-5, pp. 1069-1083, Aug. 2004.

[2] J. T. Feddema, C. R. Dohrmann, G. G. Parker, R. D. Robinett, and D. J. Schmitt, "Control for Slosh-Free Motion of an Open Container," IEEE Control Syst. Mag., vol. 17, no. 1, pp. 29-36, Feb 1997.

[3] M. Grundelius and B. Bernhardsson, "Constrained iterative learning control of liquid slosh in an industrial packaging machine," in Proc. 39th IEEE Conf. Decision and Control, Sydney, Australia, vol. 5, 2000, pp. 4544-4549.

[4] K. Yano and K. Terashima, "Robust Liquid Container Transfer Control for Complete Sloshing Suppression," IEEE Trans. Control Syst. Technol., vol. 9, no. 3, pp. 483-493, May 2001.

[5] _ - "Sloshing Suppression Control of Liquid Transfer Systems Considering a 3-D Transfer Paths," IEEE/ASME Trans. Mechatronics, vol. 10 , no. 196 , pp. 200-223, Feb. 2005.

[6] R. Venugopal and D. S. Bernstein, "State space modeling and active control of slosh," in IEEE Conf. Control Appl., vol. 4, Dearborn, MI, USA, Sept. 1996, pp. 1072-1077.

[7] P. S. Gandhi and A. Duggal, "Active stabilization of lateral and rotary slosh in cylindrical tanks," in IEEE int.Conf. Industrial Tecchnology, Feb. 2009, pp. 1-6.

[8] C. Edwards and S. K. Spurgeon, Sliding mode control: theory and application. UK: Taylor and Francis, 1998.

[9] K. J. Astrom and B. Wittenmark, Adaptive Control Second Edition. Addison-Wesley, 1995.

[10] H. Tang, L. Weng, Z. Y. Dongand, and R. Yan, "Adaptive and Learning Control for SI Engine Model With Uncertainties," IEEE/ASME Trans Mechatronics, vol. 14, no. 1, pp. 93 - 104, Feb 2009.

[11] S. Islam and P. X. Liu, "Robust Adaptive Fuzzy Output Feedback Control System for Robot Manipulators," IEEE/ASME Trans.on Mechatronics, vol. 16, no. 2, pp. 288-296, April 2011.

[12] B. Drazenovic, "The invariance conditions in variable structure systems," Automatica, vol. 5, no. 3, pp. 287-295, 1969.

[13] V. I. Utkin, "Variable structure system with sliding mode," IEEE Trans. Autom. Control, vol. 22, no. 2, pp. 212-227, April 1977.
[14] R. A. DeCarlo, S. H. Zak, and G. P. Matthews, "Variable Structure Control of Nonlinear Multivariable Systems: A Tutorial," Proc.IEEE, vol. 76, no. 3, pp. 212-232, March 1988.

[15] J. Y. Hung, W. Gao, and J. C. Hung, "Variable Structure Control: A Survey," IEEE Trans. Ind. Electron., vol. 40, no. 1, pp. 2-22, Feb. 1993.

[16] G.M.Bone and S.Ning, "Experimental Comparison of Position Tracking Control Algorithms for Pneumatic Cylinder Actuators," IEEE/ASME Trans Mechatronics, vol. 12, no. 5, pp. 557 - 561, Oct 2007.

[17] S. Bashash and N. Jalili, "Robust Adaptive Control of Coupled Parallel Piezo-Flexural Nanopositioning Stages," IEEE/ASME Trans Mechatronics, vol. 14, no. 1, pp. 11-20, Feb 2009.

[18] M. V. Pekka Ronkanen, Pasi Kallio and H. N. Koivo, "Displacement control of piezoelectric actuators using current and voltage," IEEE/ASME Trans.on Mechatronics, vol. 16, no. 1, pp. 160-166, Feb. 2011.

[19] V. F. Emiliano Pereira, Sumeet S. Aphale and S. O. R. Moheimani, "Integral Resonant Control for Vibration Damping and Precise TipPositioning of a single-link Flexible Manipulator," IEEE/ASME Trans.on Mechatronics, vol. 16, no. 2, pp. 232-240, Apr. 2011.

[20] N. Gulati and E. J. Barth, "A Globally Stable, Load-Independent Pressure Observer for the Servo Control of Pneumatic Actuators," IEEE/ASME Trans Mechatronics, vol. 14, no. 3, pp. 295 - 306, June 2009.

[21] X. Song and Z. Sun, "Pressure-Based Clutch Control for Automotive Transmissions Using a Sliding-Mode Controller," IEEE/ASME Trans.on Mechatronics to appear DOI:10.1109/TMECH.2011.2106507.

[22] Q. H. Ngo and K.-S. Hong, "Sliding-Mode Antisway Control of an Offshore Container Crane," IEEE/ASME Trans.on Mechatronics to appear DOI:10.1109/TMECH.2010.2093907.

[23] W. Wang, J. Yi, D. Zhao, and D. Liu, "Design of a stable sliding-mode controller for a class of second-order underactuated systems," IEE Proc. Control Theory and Appl., vol. 151, no. 6, p. 2004, Nov. 683- 690.

[24] M. Nikkhah, H. Ashrafiuon, and K. R. Muske, "Optimal sliding mode control for underactuated systems," in American Control Conference, USA, June 2006, pp. 4688-4693.

[25] B. L. Ma, "Comment: Design of a stable sliding-mode controller for a class of second-order underactuated systems," IET Control Theory and Appl., vol. 1, no. 4, pp. 1186-1187, Nov. 2007.

[26] B. Bandyopadhyay, S. Kurode, and P. S. Gandhi, "Sliding Mode Control for Slosh-free Motion-A Class of Underactuated System," Int. J. Adv. Mechatronic Sys., vol. 1, no. 3, pp. 203-213, Jan. 2009.

[27] R. Xu and $\ddot{U}$. Özgüner, "Sliding mode control of a class of underactuated systems," Automatica, vol. 44, no. 1, pp. 233-241, Jan. 2008.

[28] S. O. K. S. Kim, Y. Park, "Designing robust sliding hyperplanes for parametric uncertain systems: a Riccati approach," Automatica., vol. 43, pp. 1041-1048, 2004.

[29] H. H. Choi, "An Lmi based switching surface design method for a class of mismatched uncertain systems," IEEE Trans. on Autom. Control, vol. 48, pp. 1634-1638, Sept. 2003.

[30] T. Acarman and $\ddot{U}$. Özg ̈̈ner, "Rollover prevention for heavy trucks using frequency shaped sliding mode control," in Proc. IEEE Conf. Control Appl., vol. 1, June 2003, pp. 7-12.

[31] H. Richter, "Motion Control of a Container With Slosh: Constrained Sliding Mode Approach," Int. J. Dyn. Sys., Meas., Control, vol. 132, no. 3, pp. $031002-031012$, May 2010.

[32] S. Kurode, B.Bandhyopadhyay, and P. Gandhi, "Sliding Mode Control using Robust Hyperplane Design for Slosh Problem," in Proc. IEEE Int. Conf. on Avionics Systems, ICAS 2008, Hyderabad, India, Feb. 2008, pp. 101-106.

[33] S. Kurode, B. Bandyopadhyaya, and P. S. Gandhi, "Sliding mode control for slosh-free motion of a container using partial feedback linearization," in IEEE Int. workshop on variable structure systems vss-08, Antalya, Turkey, June 2008, pp. 367-372.

[34] B. Bandyopadhyay, P. S. Gandhi, and S. Kurode, "Sliding Mode Observer Based Sliding Mode Controller for Slosh-Free Motion Through PID Scheme," IEEE Trans. Ind. Electron., vol. 56, no. 9, pp. 3432-3442, Sept. 2009.

[35] S. Kurode, S. Spurgeon, B.Bandhyopadhyay, and P. Gandhi, "Sliding Mode Control for Slosh-free Motion using Nonlinear Sliding Surface," in European Control Conference ECC'09, Budapest, Hungary 2009, 2326 August 2009.

[36] R. A. Ibrahim, V. N. Pilipchuk, and T. Ikeda, "Recent advances in liquid sloshing dynamics," Applied Mechanics Review, vol. 54, no. 2, pp. 133199, 2001.

[37] D. D. Odhekar, P. S. Gandhiy, and K. B. Joshi, "Novel Methods for Slosh Parameter Estimation using Pendulum Analogy," in AIAA Atmospheric 
Flight Mechanics Conference and Exhibit, San Francisco, California., 15 - 18 Aug. 2005.

[38] H. N. Abramson, "Analytical representation of lateral sloshing by mechanical models," NASA SP-106, pp. 199-224, 1966.

[39] H. Khalil, Nonlinear systems. Upper Saddle River : Prentice Hall, 2002.

[40] P. S. Gandhi, J. Mohan, K. Joshi, and N. Anantkrishnan, "Development of 2DOF actuation slosh rig: A novel mechatronic system,", in ICIT IEEE international Conference, Bombay, India, Dec. 2006.

[41] S. Kurode, B.Bandhyopadhyay, and P. Gandhi, "Sliding mode observer for slosh-state estimation in a moving container," in Proc.IEEE int.conf. on ind.tech., Victoria, Australia., Feb. 2009, pp. 1-6.

[42] J. N. Pires, T. Godinho, and R. Arajo, "Force control for industrial applications using a fuzzy PI controller," J.:Sensor Review, vol. 24, no. 1, pp. $60-67,2004$.

[43] I. A. Mahmood, S. Moheimani, and B. Bhikkaji, "Precise Tip Positioning of a Flexible Manipulator Using Resonant Control," IEEE/ASME Trans Mechatronics, vol. 13, no. 2, pp. 180-187, April 2008.

[44] A. Fleming, "Nanopositioning System With Force Feedback for HighPerformance Tracking and Vibration Control," IEEE/ASME Trans.on Mechatronics, vol. 15, no. 3, pp. 232-240, June 2010.

[45] H. Li, M. Gong, and Z. M. Lin, "Motion Profile Design to Reduce Residual Vibration of High-Speed Positioning Stages," IEEE/ASME Trans Mechatronics, vol. 14, no. 2, pp. 264 -269, April 2009.

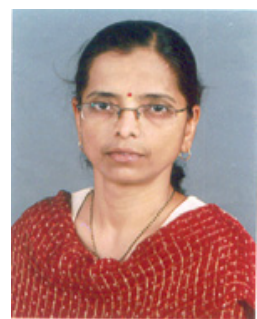

Shailaja Kurode received master's in Electrical Control Engineering, in 1998 from university of Pune, Pune, India. She received Ph.D. from Indian Institute of Technology Bombay, Mumbai in 2010. Since 1998, she has been a faculty, currently Associate Professor at College of Engineering Pune, India. She is recipient of "Rashtriya Gaurav Award 2009" from I.I.F.S. India. Her current research interests include sliding mode control for liquid fuel sloshing, discrete-time sliding mode control, Control of underactuated systems, Output feedback control. She has authored and coauthored a monograph and many refereed papers.

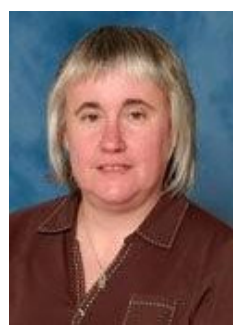

Sarha Spurgeon Sarah K Spurgeon received the B.Sc. and D.Phil. degrees from the University of York, York, U.K., in 1985 and 1988, respectively. She has held academic positions at the University of Loughborough and the University of Leicester in the UK and is currently Professor of Control Engineering and Head of the School of Engineering and Digital Arts at the University of Kent. She is a member of the Editorial Board of the International Journal of Systems Science, a member of the Editorial Board of the IET Proceedings D, a Subject Editor for the International Journal of Robust and Nonlinear Control and an Editor of the IMA Journal of Mathematical Control and Information. Her research interests are in the area of robust nonlinear control and estimation, particularly via sliding mode techniques in which area she has published in excess of 250 refereed papers. Professor Spurgeon received the IEEE Millennium Medal in 2000 and the Honeywell International Medal in 2010. She is a Fellow of the IET, a Fellow of the IMA, a Fellow of the InstMC, and was elected a Fellow of the Royal Academy of Engineering in 2008.
Bijnan Bandyopadhyay B. Bandyopadhyay received his Bachelors degree in Electronics and Telecommunication Engineering from the University of Calcutta, Calcutta, India in 1978, and Ph.D. in Electrical Engineering from the Indian Institute of Technology, Delhi, India in 1986. In 1987, he joined the Interdisciplinary Programme in Systems and Control Engineering, Indian Institute of Technology Bombay, India, as a faculty member, where he is currently a Professor. In 1996, he was with the Lehrstuhl fur Elecktrische Steuerung und Regelung, Ruhr Universitat Bochum, Bochum, Germany, as an Alexander von Humboldt Fellow. He has been a visiting Professor at Okayama University, Japan, Korea Advance Institute Science and Technology(KAIST) S.Korea and Chiba National University. He visited University of Western Australia, Australia as a Gledden Visiting Senior Fellow. He is recepient of UKIERI(UK India Education and Research Initiative) Major Award in 2007, "Distinguished Visting Fellow", 2009 award from "The Royal Academy of Engineering",London. Professor Bandyopadhyay is a Fellow of Indian National Academy of Engineering (INAE), Senior member of IEEE and a Fellow of IETE(India). He has published 7 books and monographs, 6 book chapters and more than 300 journal articles and conference papers. His research interests include the areas of Multirate output feedback control, discrete-time sliding mode control, large-scale systems, model order reduction, nuclear reactor control, smart structure control. Prof. Bandyopadhyay served as Co-Chairman of the International Organization Committee and as Chairman of the Local Arrangements Committee for the IEEE International Conference in Industrial Technology, held in Goa, India, in Jan. 2000. He also served as one of the General Chairs of IEEE ICIT conference held in Mumbai, India in December 2006. Prof. Bandyopadhyay is serving as General Chair for IEEE International Workshop on Variable Structure Systems to be held in Mumbai in January 2012.

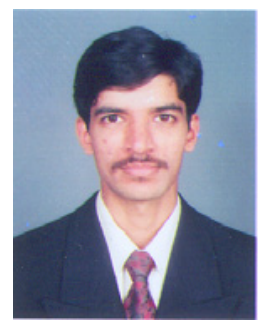

P. S. Gandhi P. S. Gandhi received the B.Eng. degree from the University of Bombay, Mumbai in 1994 and the M.Tech degree from the Indian Institute of Technology Bombay, Mumbai in 1996, both in mechanical engineering. He received the $\mathrm{Ph} . \mathrm{D}$. degree in mechanical engineering from the Rice University, Houston in 2001. Since 2001, he has been a faculty member, currently Associate Professor, in the Department of Mechanical Engineering at Indian Institute of Technology Bombay, Mumbai. His research interests are in the areas of Nonlinear Dynamical Systems and Control, Mechatronics, and MEMS. He has been a recipient of 2006 BOYSCAST fellowship to conduct research at University of California, Berkeley for six months. He has authored over 40 peer reviewed conference and journal papers, along with 1 US patent and another Indian patent. He has coordinated setup a new laboratory Suman Mashruwala Microengineering Laboratory for research in Micro-domain and has successfully completed several research projects sponsored by various Govt. research organizations. 